\title{
Percutaneous treatment of mitral regurgitation: So near, yet so far!
}

Michael J. Mack, MD

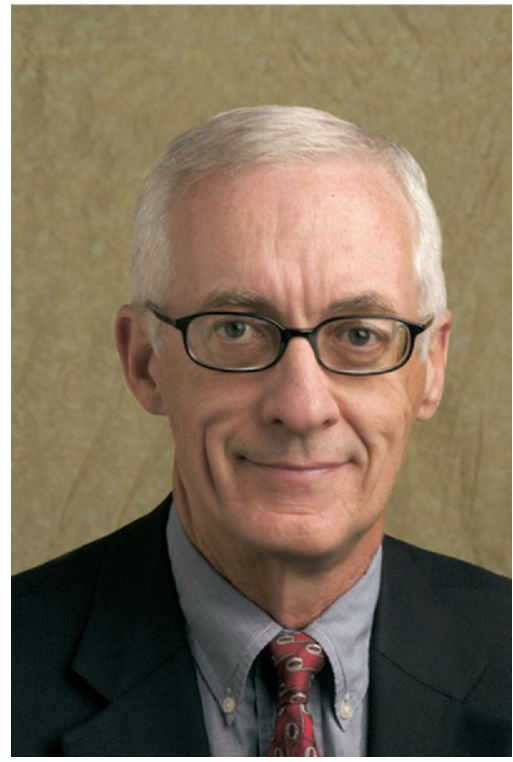

Dr Mack

See related article on page 376 .

From the Cardiopulmonary Research Science and Technology Institute, Dallas, Tex.

Michael Mack reports consulting fees from Edwards Lifesciences and Medtronic.

Received for publication June 25, 2007; accepted for publication July 9, 2007.

Address for reprints: Michael J. Mack, MD, 7777 Forest Lane, Suite A-323, Dallas, TX 75230 (E-mail: mmack@crsti.org).

J Thorac Cardiovasc Surg 2008;135:237-9

0022-5223/ $\$ 34.00$

Copyright $\odot 2008$ by The American Association for Thoracic Surgery

doi:10.1016/j.jtcvs.2007.07.063
TABLE 1. Percutaneous mitral valve approaches

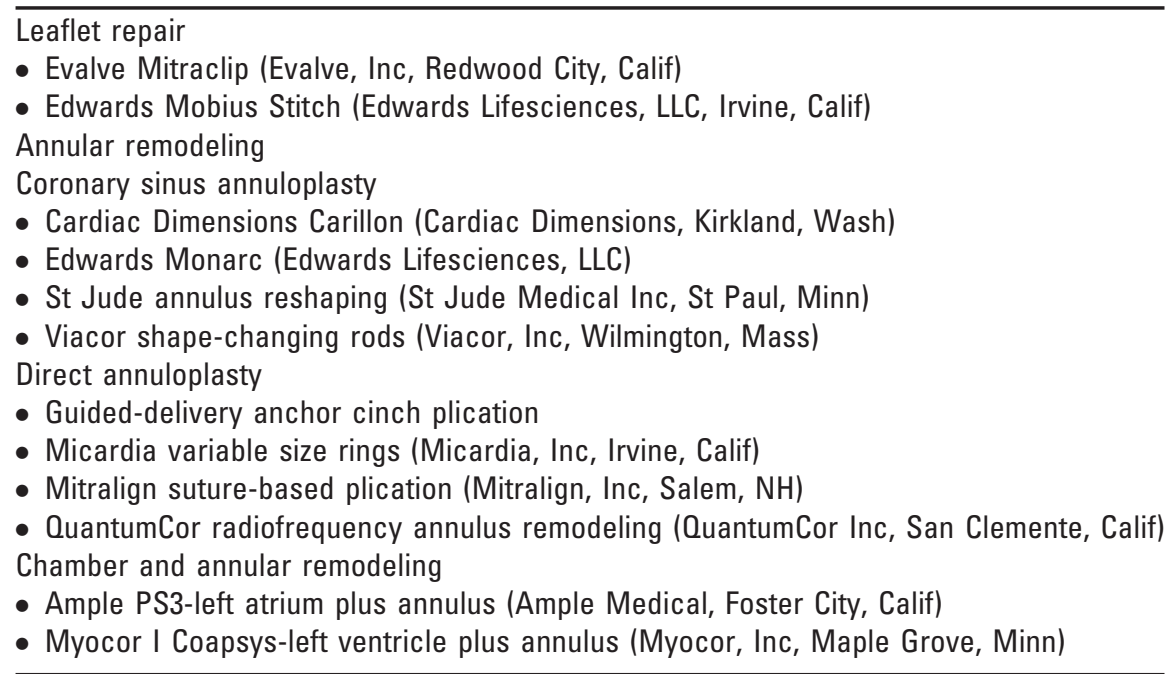

7 he percutaneous treatment of mitral regurgitation (MR) is now a clinical reality, with multiple innovative devices in proof of concept and feasibility trials and one device well into a pivotal study. More than 350 percutaneous mitral valve repair procedures have been performed with at least 6 different devices in the past 3 years. Whereas progress has been rapid and early clinical efficacy has been demonstrated in transcatheter aortic valve therapy, the demonstration of clinical benefit has been somewhat more elusive in percutaneous mitral valve therapy. This development scenario is the opposite of what was widely anticipated 5 years ago, as preclinical proof of concept studies were completed and the brave new world of clinical transcatheter valve therapy was about to commence. It seemed that that the larger clinical unmet need and greater safety margins for transcatheter mitral valve therapy would drive the clinical adoption of this technology much faster than aortic interventions. However, a number of issues have proven problematic in the road to clinical reality, including the inability to demonstrate effective proof of concept and clinical efficacy, and hurdles in regulatory trial design and patient selection.

Concepts are being used to treat MR caused by both intrinsic degenerative diseases and annular dilatation associated with functional MR (FMR) (Table 1). Percutaneous mitral valve approaches can be divided into those that address the leaflets, which are mainly but not totally being used for intrinsic disease, and those that use various concepts of annular remodeling in FMR. The Evalve Mitraclip 
(Evalve, Inc, Redwood City, Calif) device and the Edwards Mobius Stitch (Edwards Lifesciences, LLC, Irvine, Calif) device are based on the concept of the edge-to-edge leaflet approximation technique of Alfieri and colleagues. ${ }^{1}$ Some 248 patients have been enrolled in the various Evalve trials, including 55 in the EVEREST I registry, 20 in the EVEREST II high-risk registry, and the remainder in the EVEREST II pivotal trial comparing the Mitraclip in a 2:1 randomization scheme with conventional surgical mitral valve repair in patients with a lack of A2-P2 coaptation. ${ }^{2}$ Most of these patients have intrinsic degenerative disease of the leaflets, although 22 patients with FMR have been treated with this technique. One must question the inclusion of patients with FMR in this trial, because the edge-to-edge technique used surgically for functional disease has been shown to be ineffective in the surgical setting. ${ }^{3}$ Acute procedural success rate leading to less than $2+$ MR was achieved in only $61 \%$ of the first 104 patients. Seventy-three percent of patients remained surgery free at follow-up, and of those who had undergone surgery, $71 \%$ of patients were repaired and $23 \%$ of patients underwent mitral valve replacement. Despite the fact that these results of the percutaneous edge-to-edge technique do not meet the expectations of acceptable surgical results, some degree of ventricular remodeling has been reported to occur with reduced left ventricular systolic and end-diastolic dimensions at 12 months even without complete correction of the $\mathrm{MR}^{2}$ Enrollment in the pivotal trial is approximately $40 \%$ complete, and additional information to be gained from this trial includes the results of surgical mitral valve repair. Although excellent surgical results from single-center experience with $92 \%$ echocardiographic follow-up and $80 \%$ freedom from greater than 2+ MR with isolated posterior leaflet repair at 12 years have been reported, advocates of the percutaneous technique have criticized published surgical results as lacking intent-to-treat reporting, echocardiographic follow-up, and core laboratory analysis of results. This trial will indeed help to establish the true surgical benchmark. The results of the feasibility trial of the other device, the Edwards Mobius stitch, were recently presented with 6 of 15 patients improved by at least 1 MR grade at 30 days. Because of "mixed results, procedural complexity, and a perceived limited patient population, the further investigation and device development has been suspended at present." ${ }^{\text {" }}$ Whether this is an indictment of the edge-to-edge concept or merely an issue with this particular device is not clear.

FMR is a disease of the left ventricle, not of the mitral valve per se. MR in dilated cardiomyopathy is caused primarily by apical lateral distraction of the papillary muscles tethering the leaflets of the valve with secondary annular dilatation occurring the most in the septal lateral dimension. Surgical correction of FMR is based on overcorrection of the annular dilatation to reestablish an adequate surface of leaflet coaptation by a ring firmly anchored in the fibrous trigones. Percutaneous approaches for correction of FMR, with the exception of the edge-to-edge experience mentioned above, all involve some degree of annular remodeling that to varying degrees simulates surgical annuloplasty. The easy access of the coronary sinus for device delivery has proved attractive for this approach. Four devices are delivered through the coronary sinus and primarily remodel the posterior annulus to cause distraction in the septal direction. However, the article by Lansac and colleagues 5 in this issue points out some of the anatomic issues that may affect the ultimate success of mitral annuloplasty performed through the coronary sinus route. Their study on human cadaver hearts found that a coronary sinus annuloplasty at best achieves a commissure-to-commissure annuloplasty rather than being anchored in the fibrous trigones, which is an integral part of the surgical annuloplasty. In addition, they found that the coronary sinus was immediately adjacent to the left atrial wall and not the posterior mitral annulus, with an average distance between the coronary sinus and the annulus of 8 to $14 \mathrm{~mm}$ at the coronary sinus ostium, 13 to $20 \mathrm{~mm}$ in the middle of the coronary sinus, and 7 to $14 \mathrm{~mm}$ at the level of the great coronary vein. They also found that the circumflex coronary artery was located between the coronary sinus and the mitral annulus in $45 \%$ of the cases. On the basis of their anatomic study, Lansac and colleagues raised appropriate concerns about the ultimate success of coronary sinus-delivered devices. These findings were confirmed in other studies by both cadaver dissection and 3-dimensional computed tomography reconstruction. ${ }^{6-8}$ Indeed, these concerns have been realized clinically with the experience of 1 device, the CARILLON Mitral Contour System (Cardiac Dimensions, Kirkland, Wash). It was necessary to remove 2 of 34 implants because of the compromise of the coronary artery circulation resulting in ischemia. The Monarc (Edwards Lifesciences, LLC, Irvine, Calif) is a tethering device that actively foreshortens for a 6-week period of time after implantation. Early clinical results with this device and the CARILLON device are reported to show some degree of improvement of MR. Another variable compounding the placement of the coronary sinus devices is the fact that a significant number of candidate patients for these devices either have or will have coronary sinus leads for resynchronization therapy. The impact of additional hardware in the coronary sinus must be closely examined.

The coronary sinus anatomic issues have been circumvented by a number of devices that perform a direct annuloplasty. The Mitralign (Mitralign, Inc, Salem, NH) device performs a direct suture plication from the ventricular aspect while another anchor-and-cinch system also plicates the posterior annulus. The QuantumCor (QuantumCor Inc, San Clemente, Calif) device has been demonstrated in the 
animal to remodel the posterior annulus by direct application of radiofrequency energy. Two other devices remodel the mitral annulus by causing a decrease in the septal lateral dimension: the Coapsys device (Myocor, Inc, Maple Grove, Minn) from the ventricular aspect and the Ample P3 septal shortening system (Ample Medical, Foster City, Calif) via a transatrial approach by a bridge-and-tether system anchored in the coronary sinus and fossa ovalis. Early clinical proof of concept has been demonstrated with both.

Although preclinical proof of concept and clinical safety have been demonstrated with a number of devices, as well as early clinical efficacy in 2 of the coronary sinus devices, the lasting clinical benefit is of some concern. As with surgical mitral annuloplasty, it should be remembered that FMR is a disease primarily of the left ventricle and not of the mitral valve. Whereas the success of correction of MR is questionable with optimally placed surgical devices because of a high recurrence rate and difficulty demonstrating clinical benefit, the challenge to show benefit is even greater by less correction of the MR with devices delivered through either the coronary sinus or other annular remodeling approaches.

Two other intriguing options for the correction of MR worth noting include transcatheter placement of artificial chords that can be delivered through a transapical approach and an early-stage concept of transcatheter mitral valve replacement (Endovalve, Endovalve Inc, Princeton, NJ).

Although proof of concept, clinical safety, and some early clinical efficacy with percutaneous mitral devices have been demonstrated, significant challenges remain. Although the goal of all devices is the correction of MR, trial design and clinical end points are totally different for intrinsic disease and for FMR. Whereas the transcatheter aortic valve therapies are being introduced in patients with a high surgical risk or no surgical option, the trial of the edge-to-edge device is in patients who already have a good clinical option. We should keep an open mind toward new therapeutic options and await the results of the edge-to-edge pivotal trial before reaching any firm conclusions. As with any less-invasive therapy, however, the question to be answered is how much of a decrement in efficacy is acceptable for the less invasiveness of the new treatment. Given the choice, patients will uniformly choose the least-invasive option. With excellent, safe, and minimally invasive surgical approaches with proven longevity already available to treat degenerative disease of the mitral valve, the bar for clinical acceptance of catheter-based approaches should be a high one.

On the other hand, for the therapies to treat FMR where there is a huge clinical unmet need, the challenge is different. The demonstration of clinical therapeutic benefit has been difficult in optimally performed surgical annuloplasty procedures. It will be even more difficult to show therapeutic benefit by devices that of necessity do less than surgical annuloplasty. The key will be in the design of clinical trials to demonstrate efficacy where end points are likely to be functional improvement and quality of life rather than mortality. Therefore, sham or blinded trials similar to MADIT II may be necessary. There are always major hurdles to surmount in the introduction of any new therapy into clinical medicine revolving around both safety and the ability to demonstrate clinical benefit. Although we must not stifle or obstruct innovation, we also should not let unbridled enthusiasm blind ourselves to reality. The challenges of percutaneous mitral valve therapy remain formidable and clinical success is by no means assured. A balance should be struck between an open mind to new therapy and a critical eye to the early results.

\section{References}

1. Alfieri O, Maisano F, De Bonis M, Stefano PL, Torracca L, Oppizzi M, et al. The double-orifice technique in mitral valve repair: a simple solution for complex problems. J Thorac Cardiovasc Surg. 2001;122: 674-81.

2. Feldman T. Percutaneous mitral valve repair: current techniques and results. Presented at EuroPCR 2007, Barcelona, Spain. May 22-25, 2007.

3. Bhudia SK, McCarthy PM, Smedira NG, Lam BK, Rajeswaran J, Blackstone EH. Edge-to-edge (Alfieri) mitral repair: results in diverse clinical settings. Ann Thorac Surg. 2004;77:1598-606.

4. Buchbinder M. Milano II feasibility trial-Edwards' Mobius leaflet repair system. Presented at EuroPCR 2007, Barcelona, Spain. May 22-25, 2007.

5. Lansac E, Di Centa I, Attar NA, Messika-Zeitoun D, Raffoul R, Vahanian A, et al. Percutaneous mitral annuloplasty through the coronary sinus: an anatomical point of view. J Thorac Cardiovasc Surg. 2007; 8:376-81.

6. Choure AJ, Garcia MJ, Hesse B, Sevensma M, Maly G, Greenberg NL, et al. In vivo analysis of the anatomical relationship of coronary sinus to mitral annulus and left circumflex coronary artery using cardiac multidetector computer tomography: implications for percutaneous coronary sinus mitral annuloplasty. J Am Coll Cardiol. 2006;48:1938-45.

7. Tops LF, Van de Veire NR, Schuijf JD, de Roos A, van der Wall EE, Schalij MJ, et al. Noninvasive evaluation of coronary sinus anatomy and its relation to the mitral valve annulus: implications for percutaneous annuloplasty. Circulation. 2007;115:1426-32.

8. Maselli D, Guarracino F, Chiaramonti F, Mangia F, Borelli G, Minzioni G. Percutaneous mitral annuloplasty: an anatomic study of human coronary sinus and its relation with mitral valve annulus and coronary arteries. Circulation. 2006;114:377-80. 